\title{
Morfometria do intestino delgado de frangos tratados com dietas adicionadas de mananoligossacarídeo e complexo enzimático
}

\author{
Maria Cristina de Oliveira ${ }^{1 *}$ \\ Rafael Henrique Marques ${ }^{2}$ \\ Rodrigo Antônio Gravena ${ }^{2}$ \\ Vera Maria Barbosa de Moraes ${ }^{2}$ \\ ${ }^{1}$ Faculdade de Medicina Veterinária, Universidade de Rio Verde \\ Caixa Postal 244 \\ CEP 75901-970, Rio Verde - GO, Brasil \\ ${ }^{2}$ Departamento de Zootecnia, FCAV/UNESP, Jaboticabal - SP, Brasil \\ *Autor para correspondência \\ cristina@fesurv.br
}

Submetido em 29/11/2007

Aceito para publicação em 27/05/2008

\section{Resumo}

Estudou-se o efeito do mananoligossacarídeo (MOS) e de complexo enzimático (CE) sobre a morfometria do intestino delgado de frangos. Foram utilizadas 750 aves em delineamento inteiramente casualizado e arranjo fatorial 2 × $2+1$ - dois níveis de $\operatorname{MOS}(0$ e $0,1 \%$ de 1 a 21 dias e $0,05 \%$ de 22 a 42 dias de idade), dois níveis de CE ( 0 e $0,05 \%)$ e a dieta controle positivo com antibióticos - totalizando cinco tratamentos e cinco repetições. A interação antibiótico x fatorial foi significativa para espessura da muscular longitudinal e para superfície de absorção no duodeno e no íleo, sendo maior nas aves do tratamento com MOS. A interação MOS x CE foi significativa para mucosa do duodeno aos 21 dias e jejuno e íleo aos 42 dias, sendo menos espessa nas aves do tratamento sem aditivos. $\mathrm{O}$ mesmo efeito foi observado para superfície de absorção duodenal aos 21 dias e no íleo aos 42 dias. A inclusão de MOS nas dietas aumentou $(\mathrm{P}<0,05)$, e de enzimas diminuiu $(\mathrm{P}<0,02)$, a espessura da camada muscular longitudinal do duodeno aos 42 dias. A adição de MOS às dietas aumentou as espessuras da muscular longitudinal e da mucosa, o que se refletiu em maior superfície de absorção intestinal.

Unitermos: aditivos, celulase, nutrição animal, prebiótico, protease

\section{Abstract}

Morphometry of the small intestine from broilers treated with diets added of mannan oligosaccharides and enzymatic complex. The effects of mannan oligosaccharides (MOS) and enzimatic complex (EC) on the morphometry of the small intestine wall in broilers were studied. Seven hundred and fifty birds were used in a completely randomized design and $2 \times 2+1$ factorial arrangement - two MOS levels $(0$ and $0.1 \%$ from 1 to 21 days and $0.05 \%$ from 22 to 42 days of age), two EC levels ( 0 and $0.05 \%$ ) and a positive control diet with antibiotics - totaling five treatments with five replicates. Positive control $\mathrm{x}$ factorial interaction was significant for the longitudinal muscular thickness and for the absorption surface in the duodenum and ileum, being higher in birds subjected to MOS treatment. MOS x EC interaction was significant for the duodenal mucosa at 21 days and for the jejunum and ileum at 42 days, being lower in birds treated with no additives at all. The same effect was absorved duodenal absorption surface at 21 days, and for the ileum at 42 days. MOS 
inclusion increased $(\mathrm{P}<0.05)$, and $\mathrm{EC}$ inclusion reduced $(\mathrm{P}<0.02)$, the longitudinal muscular thickness of the duodenum at 42 days. MOS addition increased the longitudinal muscular thickness and the mucosa, and this was reflected in the form of a more extensive intestinal absorption surface.

Key words: additives, animal nutrition, cellulase, prebiotics, protease

\section{Introdução}

Após o nascimento, o intestino delgado das aves continua a crescer em peso mais rapidamente do que a massa corporal total. Este crescimento relativo do intestino delgado é máximo entre seis e 10 dias de idade nos frangos e ocorre na presença ou ausência de alimentos (Mateos et al., 2004; Sklan, 2004), porém, a ingestão de alimentos estimula o desenvolvimento do trato gastrintestinal (TGI) (Gracia et al., 2003) sendo que o crescimento do duodeno ocorre mais cedo do que do jejuno e do íleo (Uni et al., 1999).

O elemento funcional do intestino delgado é a mucosa, que pode ser caracterizada como uma camada permeável a nutrientes e barreira contra compostos nocivos. A competição entre bactérias e hospedeiro por nutrientes e a formação de metabólitos depressores do crescimento no intestino podem ter efeitos negativos sobre a mucosa do intestino delgado. Acredita-se que a inibição da atividade microbiana pelos antibióticos reduz a competição com o hospedeiro pelos nutrientes e a produção de metabólitos depressores do crescimento (van Leeuwen, 2002).

A adição de antibióticos ao alimento em baixos níveis é prática comum na produção de aves e melhora o ganho de peso e a eficiência alimentar em torno de um a 5\% (Mateos et al., 2004). Os antibióticos controlam e limitam não só o crescimento de microrganismos prejudiciais às aves, mas também de bactérias não-patogênicas incluindo lactobacilos, bifidobactérias, bacteróides e enterococos, reduzem a produção de antagonistas microbianos como a amônia que afeta adversamente a fisiologia do hospedeiro (Ferket, 2002).

A microflora do TGI desempenha importante papel na manutenção da saúde do hospedeiro. Os benefícios da ingestão de prebióticos incluem aumento da população de bifidobactérias e lactobacilos nativos que suprimem a atividade de bactérias putrefativas e reduzem a formação de produtos tóxicos da fermentação (Flickinger et al., 2003). Dentre os prebióticos se destaca o mananoligossacarídeo (MOS) derivado da parede celular de Saccharomyces cerevisiae, comercialmente disponível como suplemento alimentar. Seus benefícios são baseados na modificação da flora intestinal e modulação do sistema imune intestinal (Shane, 2001).

As fibras consistem de todos os polissacarídeos não-amiláceos (PNAs) resistentes à digestão no intestino delgado e fermentáveis no intestino grosso, onde microrganismos produzem alguns tipos de toxinas. Estas toxinas causam lesões no epitélio da mucosa e contribuem para o espessamento desta camada (Jacobs e Lupton, 1984) e causam danos mecânicos ao epitélio (Chiou et al., 1994). Exemplos destes PNAs incluem celulose, açúcares não-glicosídicos, açúcares ácidos, gomas e mucilagens (Tungland e Meyer, 2002). Embora dietas a base de milho e farelo de soja não sejam consideradas de alta viscosidade, os dois ingredientes possuem PNAs em suas estruturas. O milho contém, aproximadamente, $0,9 \%$ de PNAs solúveis e $6 \%$ insolúveis enquanto que o farelo de soja contém, aproximadamente, $6 \%$ de PNAs solúveis e 18 a $20 \%$ de insolúveis (Kocher et al., 2003). O amido não-digerido no íleo terminal é denominado de amido resistente e se apresenta como uma oportunidade para ser degradado pelas enzimas microbianas suplementadas nas dietas (Iji et al., 2003). A inclusão de enzimas que degradem a celulose e o amido seria ferramenta útil para aumentar o teor de energia das dietas e melhorar o desempenho das aves.

Avaliaram-se os efeitos da inclusão de MOS e/ou complexo enzimático (CE) na dieta de frangos de corte sobre a morfometria da parede intestinal.

\section{Material e Métodos}

Foram utilizados 750 pintos, de um dia, linhagem Cobb e peso inicial médio de $41,51 \pm 0,59 \mathrm{~g}$. As aves 
foram alojadas em boxes experimentais com 30 aves cada. O delineamento foi inteiramente casualizado em arranjo fatorial $2 \times 2+1 \mathrm{com}$ dois níveis de $\mathrm{MOS}^{1}(0 \mathrm{e}$ $0,1 \%$ de 1 a 21 dias e $0,05 \%$ de 22 a 42 dias de idade), dois níveis de $\mathrm{CE}^{1}(0$ e $0,05 \%)$ e uma dieta controle positivo (125ppm de sulfato de colistina e $10 \mathrm{ppm}$ de virginiamicina), totalizando cinco tratamentos e cinco repetições. A dieta sem antibióticos, MOS e CE, foi considerada como dieta controle negativo.

A fitase, com atividade mínima de $250 \mathrm{U} / \mathrm{g}$, foi utilizada em todas as dietas e o nível de fósforo não-fítico utilizado foi de $0,38 \%$, correspondendo a uma redução de $85 \%$ nas exigências da ave. O CE na forma líquida, era composto de celulase, protease e $\alpha$-amilase. Ambos foram misturados às dietas de acordo com instruções dos fabricantes sendo que $\mathrm{O}$ CE foi pré-misturado a $500 \mathrm{~g}$ de farelo de soja antes dos ingredientes irem para o misturador.
As dietas experimentais (Tabela 1) foram formuladas de acordo com Rostagno et al. (2000) e eram isonutritivas, exceto pela energia metabolizável e pela proteína bruta que corresponderam a $98 \%$ dos níveis nutricionais descritos pelos mesmos autores.

Após 12 horas de jejum alimentar, cinco aves por tratamento foram sacrificadas por deslocamento cervical, aos 21 e 42 dias de idade. Fragmentos de $2 \mathrm{~cm}$. retirados da metade do comprimento da alça duodenal, aos $10 \mathrm{~cm}$ acima do divertículo de Meckel (jejuno) e $10 \mathrm{~cm}$ acima da junção ileocecal (íleo) de cada ave, foram imersos em solução fixadora de Bouin durante 24 horas. Em seguida, os fragmentos foram processados para inclusão em parafina. Os cortes seriados de $5 \mu \mathrm{m}$. realizados em cada fragmento, foram corados com hematoxilina e eosina.

TABELA 1: Composição das dietas experimentais.

\begin{tabular}{|c|c|c|c|c|c|c|c|c|c|c|}
\hline \multirow[b]{2}{*}{ Ingredientes } & \multicolumn{5}{|c|}{1 a 21 dias } & \multicolumn{5}{|c|}{22 a 42 dias } \\
\hline & $\begin{array}{c}\text { Controle } \\
\text { positivo }\end{array}$ & $\begin{array}{l}\text { Controle } \\
\text { negativo }\end{array}$ & CE & MOS & $\begin{array}{c}\text { CE } \\
+ \text { MOS }\end{array}$ & $\begin{array}{c}\text { Controle } \\
\text { positivo }\end{array}$ & $\begin{array}{l}\text { Controle } \\
\text { negativo }\end{array}$ & CE & MOS & $\begin{aligned} & \text { CE } \\
+ & \text { MOS }\end{aligned}$ \\
\hline Milho moído & 59,74 & 59,74 & 59,74 & 59,74 & 59,74 & 65,69 & 65,69 & 65,69 & 65,69 & 65,69 \\
\hline Farelo de soja & 34,34 & 34,34 & 34,34 & 34,34 & 34,34 & 28,62 & 28,62 & 28,62 & 28,62 & 28,62 \\
\hline Óleo de soja & 1,58 & 1,58 & 1,58 & 1,58 & 1,58 & 1,87 & 1,87 & 1,87 & 1,87 & 1,87 \\
\hline Fosfato bicálcico & 1,45 & 1,45 & 1,45 & 1,45 & 1,45 & 1,26 & 1,26 & 1,26 & 1,26 & 1,26 \\
\hline Calcário calcítico & 1,22 & 1,22 & 1,22 & 1,22 & 1,22 & 1,17 & 1,17 & 1,17 & 1,17 & 1,17 \\
\hline DL-Metionina 99\% & 0,04 & 0,04 & 0,04 & 0,04 & 0,04 & 0,03 & 0,03 & 0,03 & 0,03 & 0,03 \\
\hline L-Lisina $78,80 \%$ & 0,20 & 0,20 & 0,20 & 0,20 & 0,20 & 0,25 & 0,25 & 0,25 & 0,25 & 0,25 \\
\hline Sal comum & 0,45 & 0,45 & 0,45 & 0,45 & 0,45 & 0,38 & 0,38 & 0,38 & 0,38 & 0,38 \\
\hline Fitase & 0,01 & 0,01 & 0,01 & 0,01 & 0,01 & 0,01 & 0,01 & 0,01 & 0,01 & 0,01 \\
\hline MOS & - & - & - & 0,10 & 0,10 & - & - & - & 0,05 & 0,05 \\
\hline $\mathrm{CE}$ & - & - & 0,05 & - & 0,05 & - & - & 0,05 & - & 0,05 \\
\hline Caulim & 0,15 & 0,15 & 0,10 & 0,05 & - & 0,10 & 0,10 & 0,05 & 0,05 & - \\
\hline BHT & 0,02 & 0,02 & 0,02 & 0,02 & 0,02 & 0,02 & 0,02 & 0,02 & 0,02 & 0,02 \\
\hline \multirow[t]{2}{*}{ Supl. mineral/vitamínico } & $0,80^{1}$ & $0,80^{2}$ & $0,80^{2}$ & $0,80^{2}$ & $0,80^{2}$ & $0,60^{3}$ & $0,60^{4}$ & $0,60^{4}$ & $0,60^{4}$ & $0,60^{4}$ \\
\hline & \multicolumn{10}{|c|}{ Composição calculada } \\
\hline Proteína bruta (\%) & 21,00 & 21,00 & 21,00 & 21,00 & 21,00 & 18,92 & 18,92 & 18,92 & 18,92 & 18,92 \\
\hline $\mathrm{EM}(\mathrm{kcal} / \mathrm{kg})$ & 2940 & 2940 & 2940 & 2940 & 2940 & 3038 & 3038 & 3038 & 3038 & 3038 \\
\hline Cálcio $(\%)$ & 0,96 & 0,96 & 0,96 & 0,96 & 0,96 & 0,87 & 0,87 & 0,87 & 0,87 & 0,87 \\
\hline Fósforo disponível (\%) & 0,38 & 0,38 & 0,38 & 0,38 & 0,38 & 0,34 & 0,34 & 0,34 & 0,34 & 0,34 \\
\hline Lisina $(\%)$ & 1,26 & 1,26 & 1,26 & 1,26 & 1,26 & 1,16 & 1,16 & 1,16 & 1,16 & 1,16 \\
\hline Metionina $(\%)$ & 0,49 & 0,49 & 0,49 & 0,49 & 0,49 & 0,45 & 0,45 & 0,45 & 0,45 & 0,45 \\
\hline
\end{tabular}

${ }^{1}$ cada kg contém: 1500000 UI Vit. A, 285000 UI Vit.D, $1350 \mathrm{mg}$ Vit.E, $230 \mathrm{mg}$ Vit.K, $115 \mathrm{mg}$ Vit.B, $1150 \mathrm{mg}$ Vit.B, 2000 mcg Vit.B ${ }_{12}, 4800$ $\mathrm{mg}$ ácido nicotínico, $1240 \mathrm{mg}$ ácido pantotênico, $230 \mathrm{mg}$ piridoxina, $12 \mathrm{mg}$ biotina, $115 \mathrm{mg}$ ácido fólico, $85 \mathrm{~g}$ colina, $170 \mathrm{~g}$ metionina, 6300 $\mathrm{mg} \mathrm{Fe}, 9400 \mathrm{mg} \mathrm{Cu}, 9400 \mathrm{mg} \mathrm{Mn}, 7819 \mathrm{mg} \mathrm{Zn}, 160 \mathrm{mg} \mathrm{I}, 23 \mathrm{mg} \mathrm{Se}, 20 \mathrm{~g}$ antioxidante, 5,4 g promotor de crescimento, 6,4 g anticoccidiano. 2 idem 1, exceto pelo promotor de crescimento e anticoccidiano. ${ }^{3}$ cada kg contém: 1550000 UI Vit.A, 267000 UI Vit.D, 1200 mg Vit.E, 200 mg Vit. $\mathrm{K}_{3}, 100 \mathrm{mg}$ Vit. $\mathrm{B}_{1}, 935 \mathrm{mg}$ Vit. $\mathrm{B}_{2}, 2340 \mathrm{mcg}$ Vit. $\mathrm{B}_{12}, 4700 \mathrm{mg}$ ácido nicotínico, $1340 \mathrm{mg}$ ácido pantotênico, $170 \mathrm{mg}$ piridoxina, $14 \mathrm{mg}$ biotina, $100 \mathrm{mg}$ ácido fólico, $84 \mathrm{~g}$ colina, $185 \mathrm{~g}$ metionina, $6720 \mathrm{mg} \mathrm{Fe}, 10000 \mathrm{mg} \mathrm{Cu}, 10200 \mathrm{mg} \mathrm{Mn}, 8418 \mathrm{mg} \mathrm{Zn,} 170 \mathrm{mg} \mathrm{I}, 24 \mathrm{mg} \mathrm{Se,} 20 \mathrm{~g}$ antioxidante, $8,5 \mathrm{~g}$ promotor de crescimento, $8,5 \mathrm{~g}$ anticoccidiano. ${ }^{4}$ idem 3 , exceto pelo promotor de crescimento e anticoccidiano.

1 Alltech Agroindustrial do Brasil Ltda. Araucária, PR, Brasil. 
Foram realizadas 40 leituras por fragmento para espessura das camadas mucosa (EM), submucosa (ESM), muscular circular (EMC) e muscular longitudinal (EML). A superfície de absorção (SA) foi calculada de acordo com a fórmula (Hardin et al., 1999):

\section{SA $\left(\mathrm{mm}^{2}\right)=$ altura do vilo $(\mathrm{mm}) \times$ largura a $50 \%$ de altura do vilo $(\mathrm{mm})$}

Os resultados obtidos foram submetidos à ANOVA sendo que as médias do fatorial MOS x CE foram comparadas utilizando-se o teste de Fischer e, para comparar as médias do controle positivo $\mathrm{x}$ fatorial, foi utilizado o teste Dunnett (Statsoft, 1995).

\section{Resultados e Discussão}

Não houve efeito $(\mathrm{P}>0,05)$ das interações controle positivo $\mathrm{x}$ fatorial e MOS $\mathrm{x}$ CE sobre ESM e EMC em todos os segmentos, da EM no duodeno (Tabela 2) aos 42 dias e no jejuno (Tabela 3) e íleo (Tabela 4) aos 21 dias, da EML no duodeno aos 21 dias e no jejuno e íleo aos 21 e 42 dias e sobre a SA no duodeno aos 42 dias, jejuno aos 21 e 42 dias e no íleo aos 21 dias de idade.

A interação MOS x CE foi significativa para EM no duodeno $(\mathrm{P}<0,01)$ aos 21 dias e no jejuno $(\mathrm{P}<0,02)$ (Tabela 2) e íleo $(\mathrm{P}<0,01)$ (Tabela 3 ) aos 42 dias. A mucosa das aves que ingeriram dietas sem aditivos era mais fina. No íleo, entretanto, as aves do tratamento com MOS + CE também apresentaram a mucosa mais fina. Os AGCC, produzidos por meio da fermentação microbiana do MOS, são eficazes em estimular o desenvolvimento da mucosa intestinal porque servem de fonte de energia para os enterócitos (Ferket, 2004). Já o CE desempenha um papel estimulatório da mucosa intestinal por reduzir a quantidade de substrato disponível para degradação bacteriana. Pelo exposto acredita-se que as aves que ingeriram dietas sem aditivos (controle negativo) tiveram, possivelmente, maior carga bacteriana no intestino, o que pode ter causado irritação e danos à mucosa (Brenes et al., 2002). No íleo, entretanto, a espessura da mucosa diminuiu quando o MOS e o CE foram adicionados às dietas, provavelmente, devido ao aumento da digestibilidade dos nutrientes nesta região do intestino causado pela associação dos dois aditivos (Oliveira et al., 2007).
A interação antibiótico $\mathrm{x}$ fatorial foi significativa $(\mathrm{P}<0,05)$ para $\mathrm{EML}$ no duodeno aos 42 dias de idade. A EML das aves que não receberam somente $\mathrm{CE}$ em suas dietas apresentou-se mais delgada do que a das aves do controle positivo. A inclusão do MOS às dietas aumentou $(\mathrm{P}<0,05)$ a EML duodenal aos 21 e 42 dias, enquanto que a inclusão de $\mathrm{CE}$ reduziu $(\mathrm{P}<0,02)$ esse parâmetro aos 42 dias. Os antibióticos agem tanto em bactérias patogênicas quanto sobre as benéficas (Ferket et al., 2002) diminuindo, assim, a população bacteriana em geral. As enzimas também são úteis na redução da carga bacteriana, uma vez que a hidrólise dos PNAs diminui a quantidade de substratos disponíveis para a proliferação das bactérias (Santos Jr. et al., 2004). É provável que a adição de antibióticos ou $\mathrm{CE}$ às dietas tenha causado a diminuição da produção microbiana de poliaminas e ácidos graxos de cadeia curta (AGCC), conhecidos por aumentar a atividade celular (van Leeuwen, 2002). A hidrólise dos PNAs, amido e proteínas da dieta pode resultar em menor quantidade e peso de digesta, causando afinamento da camada muscular longitudinal (Brenes et al., 2002), que é uma das camadas responsáveis pela peristalse. Este mesmo efeito foi constatado também por McDonald et al. (2001). Já os efeitos estimulatórios do MOS se deram por meio da produção de ácidos graxos de cadeia curta após a sua fermentação no intestino e esses ácidos aceleram a proliferação celular no epitélio intestinal (Oliveira et al., 2008).

Schwarz et al. (2002), porém, testaram dietas contendo avilamicina ou MOS e relataram não haver diferença na espessura da camada muscular da parede intestinal dos frangos em função dos tratamentos. No que se refere à ação das enzimas, Brenes et al. (2002) avaliaram três complexos multienzimáticos em dietas a base de tremoço e notaram que os pesos relativos do duodeno, jejuno e íleo foram menores nas aves alimentadas com dietas contendo um ou a mistura dos complexos enzimáticos. Os autores associaram este fato à redução no número de bactérias presentes no intestino delgado, devido à redução na quantidade de substratos disponíveis para as bactérias.

Como já mencionado, os antibióticos limitam o crescimento e a colonização de bactérias no intestino (Ferket et al., 2002; van Leeuwen, 2002). A redução de 
bactérias, como lactobacilos e bifidobactérias, diminui a produção de $\mathrm{AGCC}$ e, conseqüentemente, o pH intestinal aumenta, favorecendo o desenvolvimento de bactérias patogênicas (Edens et al., 1997), que danificam a mucosa intestinal (Ferket, 2004). O MOS reduz a presença de patógenos no intestino (Mathew, 2001) e aumenta a produção de AGCC após sua fermentação e estes ácidos estão associados à proliferação celular no epitélio intestinal, aumentando a altura e o perímetro dos vilos e também a área de absorção (Oliveira et al., 2008).

A interação antibiótico $\mathrm{x}$ fatorial foi significativa para SA $(\mathrm{P}<0,01)$ no duodeno e no íleo aos 21 e 42 dias, respectivamente. A SA nas aves que receberam dietas contendo somente MOS foi maior do que nas aves que receberam dietas com antibióticos como promotores do crescimento.
Houve efeito também da interação MOS x CE para área de superfície de absorção em que no duodeno $(\mathrm{P}<0,0)$ aos 21 dias e no íleo $(\mathrm{P}<0,0)$ aos 42 dias, a área de absorção foi menor nas aves submetidas à dietas sem aditivos. No entanto, as aves do tratamento com MOS + CE também apresentaram menor SA, tanto no duodeno quanto no íleo. A menor área de absorção de aves submetidas a dietas sem aditivos ocorreu devido à maior proliferação bacteriana no intestino. $\mathrm{O}$ excesso de bactérias causa irritação e danos à mucosa intestinal além de diminuir a quantidade de nutrientes disponíveis para absorção. A redução da área de absorção em aves dos tratamentos com MOS + CE se deve à maior digestibilidade de nutrientes o que diminuiu a demanda por aumento do tecido intestinal (Gilbert et al., 2001).

TABELA 2: Morfometria da parede do duodeno de frangos de corte, alimentados com dietas contendo MOS e/ou complexo enzimático (CE).

\begin{tabular}{|c|c|c|c|c|c|c|c|c|c|c|c|c|}
\hline \multirow{3}{*}{ Parâmetros } & \multicolumn{6}{|c|}{21 dias } & \multicolumn{6}{|c|}{42 dias } \\
\hline & \multirow{2}{*}{$\mathrm{CP}$} & \multirow{2}{*}{ MOS } & \multicolumn{2}{|c|}{ CE } & \multirow{2}{*}{ Média } & \multirow{2}{*}{$\begin{array}{l}\text { CV } \\
(\%)\end{array}$} & \multirow{2}{*}{$\mathbf{C P}$} & \multirow{2}{*}{ MOS } & \multicolumn{2}{|c|}{$\mathrm{CE}$} & \multirow{2}{*}{-Média } & \multirow{2}{*}{$\begin{array}{l}\text { CV } \\
(\%) \\
\end{array}$} \\
\hline & & & Sem & Com & & & & & Sem & Com & & \\
\hline \multirow{3}{*}{$\operatorname{Mucosa}(\mu \mathrm{m})$} & & Sem & $1619 \mathrm{Bb}$ & 1769Aa & 1694 & \multirow[b]{3}{*}{5,86} & \multirow[b]{3}{*}{1837} & Sem & 1825 & 1850 & 1838 & \\
\hline & & Com & $1836 A a$ & 1730Aa & 1783 & & & Com & 1925 & 1871 & 1898 & \\
\hline & 1700 & Média & 1728 & 1749 & & & & Média & 1875 & 1861 & & 4,26 \\
\hline \multirow{3}{*}{ Submucosa $(\mu \mathrm{m})$} & & Sem & 36 & 44 & 40 & & \multirow[b]{3}{*}{49} & Sem & 46 & 43 & 45 & \\
\hline & & Com & 37 & 40 & 39 & & & Com & 43 & 44 & 44 & \\
\hline & 40 & Média & 37 & 42 & & 8,13 & & Média & 45 & 44 & & 5,52 \\
\hline \multirow{3}{*}{ Muscular circular $(\mu \mathrm{m})$} & & Sem & 234 & 210 & 222 & & \multirow[b]{3}{*}{255} & Sem & 273 & 252 & 263 & \\
\hline & & Com & 250 & 198 & 224 & & & Com & 270 & 273 & 272 & \\
\hline & 227 & Média & 242 & 204 & & 5,26 & & Média & 272 & 263 & & 3,45 \\
\hline \multirow{3}{*}{ Muscular longitudinal $(\mu \mathrm{m})$} & & Sem & 66 & 72 & $69 \mathrm{~A}$ & \multirow[b]{3}{*}{9,05} & \multirow[b]{3}{*}{62} & Sem & $91^{*}$ & 56 & 74B & \multirow[b]{3}{*}{8,52} \\
\hline & & Com & 50 & 59 & $54 \mathrm{~B}$ & & & Com & $94 *$ & $88 *$ & $91 \mathrm{~A}$ & \\
\hline & 61 & Média & 58 & 65 & & & & Média & $93 a$ & $72 b$ & & \\
\hline \multirow{3}{*}{ Superfície de absorção $\left(\mathrm{mm}^{2}\right)$} & & Sem & $0,095 \mathrm{Bb}$ & $0,118 \mathrm{Aa}$ & 0,107 & \multirow[b]{3}{*}{7,30} & \multirow[b]{3}{*}{0,133} & Sem & 0,124 & 0,137 & 0,131 & \\
\hline & & Com & $0,128 \mathrm{Aa}^{*}$ & $0,114 \mathrm{Aa}$ & 0,121 & & & Com & 0,125 & 0,141 & 0,133 & \multirow[b]{2}{*}{11,31} \\
\hline & 0,095 & Média & 0,112 & 0,116 & & & & Média & 0,124 & 0,139 & & \\
\hline
\end{tabular}

$\mathrm{CP}=$ controle positivo.

$\mathrm{CV}=$ coeficiente de variação obtido com médias transformadas (log X).

* Média diferente da média do tratamento com antibióticos pelo teste Tukey.

Médias seguidas de letras diferentes maiúsculas nas colunas e minúsculas nas linhas, diferem entre si pelo teste Tukey. 
TABELA 3: Morfometria da parede do jejuno de frangos de corte aos 21 e 42 dias de idade, alimentados com dietas contendo MOS e/ou complexo enzimático (CE).

\begin{tabular}{|c|c|c|c|c|c|c|c|c|c|c|c|c|}
\hline \multirow{3}{*}{ Parâmetros } & \multicolumn{4}{|c|}{21 dias } & \multirow{3}{*}{ Média } & \multirow{3}{*}{$\begin{array}{l}\text { CV } \\
(\%)\end{array}$} & \multirow{3}{*}{ CP } & \multirow{3}{*}{ MOS } & \multicolumn{4}{|c|}{42 dias } \\
\hline & \multirow{2}{*}{ CP } & \multirow{2}{*}{ MOS } & \multicolumn{2}{|c|}{ CE } & & & & & \multicolumn{2}{|c|}{ CE } & \multirow{2}{*}{ Média } & \multirow{2}{*}{$\begin{array}{l}\text { CV } \\
(\%)\end{array}$} \\
\hline & & & Sem & Com & & & & & Sem & Com & & \\
\hline \multirow{3}{*}{ Mucosa $(\mu \mathrm{m})$} & & Sem & 978 & 1078 & 1028 & & & Sem & $1407 \mathrm{Bb}$ & 1658Aa & 1533 & \\
\hline & & Com & 1161 & 1120 & 1140 & \multirow[b]{2}{*}{1,95} & \multirow[b]{2}{*}{1442} & Com & $1627 \mathrm{Aa}$ & $1514 \mathrm{Aa}$ & 1570 & \\
\hline & 1140 & Média & 1069 & 1099 & & & & Média & 1517 & 1586 & 0,02 & 4,98 \\
\hline \multirow{3}{*}{ Submucosa $(\mu \mathrm{m})$} & & Sem & 45 & 34 & 39 & \multirow[b]{3}{*}{4,29} & \multirow[b]{3}{*}{51} & Sem & 63 & 60 & 62 & \\
\hline & & Com & 39 & 37 & 38 & & & Com & 58 & 56 & 57 & \\
\hline & 45 & Média & 42 & 35 & & & & Média & 60 & 58 & & 3,93 \\
\hline \multirow{3}{*}{ Muscular circular $(\mu \mathrm{m})$} & & Sem & 156 & 117 & 136 & \multirow[b]{3}{*}{5,89} & \multirow[b]{3}{*}{174} & Sem & 209 & 257 & 233 & \\
\hline & & Com & 119 & 114 & 117 & & & Com & 242 & 216 & 229 & \\
\hline & 145 & Média & 137 & 116 & & & & Média & 226 & 236 & & 4,40 \\
\hline \multirow{3}{*}{ Muscular longitudinal $(\mu \mathrm{m})$} & & Sem & 66 & 39 & 52 & \multirow[b]{3}{*}{7,67} & \multirow[b]{3}{*}{67} & Sem & 71 & 70 & 70 & \multirow[b]{3}{*}{6,89} \\
\hline & & Com & 43 & 42 & 42 & & & Com & 86 & 78 & 82 & \\
\hline & 56 & Média & 54 & 40 & & & & Média & 78 & 74 & & \\
\hline \multirow{3}{*}{ Superfície de absorção $\left(\mathrm{mm}^{2}\right)$} & & Sem & 0,058 & 0,064 & 0,061 & \multirow[b]{3}{*}{16,33} & \multirow[b]{3}{*}{0,084} & Sem & 0,081 & 0,106 & 0,094 & \\
\hline & & Com & 0,058 & 0,065 & 0,062 & & & Com & 0,088 & 0,098 & 0,093 & \multirow[b]{2}{*}{17,86} \\
\hline & 0,060 & Média & 0,058 & 0,065 & & & & Média & $0,085 b$ & $0,102 \mathrm{a}$ & & \\
\hline
\end{tabular}

$\mathrm{CP}=$ controle positivo.

$\mathrm{CV}=$ coeficiente de variação obtido com médias transformadas $(\log \mathrm{X})$.

Médias seguidas por letras diferentes maiúsculas nas colunas e minúsculas nas linhas diferem entre si pelo teste Tukey.

TABELA 4: Morfometria da parede do íleo de frangos de corte aos 21 e 42 dias de idade, alimentados com dietas contendo MOS e/ou complexo enzimático (CE).

\begin{tabular}{|c|c|c|c|c|c|c|c|c|c|c|c|c|}
\hline \multirow{3}{*}{ Parâmetros } & \multicolumn{6}{|c|}{21 dias } & \multicolumn{6}{|c|}{42 dias } \\
\hline & \multirow{2}{*}{ CP } & \multirow{2}{*}{ MOS } & \multicolumn{2}{|c|}{ CE } & \multirow{2}{*}{ - Média } & \multirow{2}{*}{$\begin{array}{l}\text { CV } \\
(\%)\end{array}$} & \multirow{2}{*}{$\mathbf{C P}$} & \multirow{2}{*}{ MOS } & \multicolumn{2}{|c|}{ CE } & \multirow{2}{*}{ Média } & \multirow{2}{*}{$\begin{array}{l}\text { CV } \\
(\%)\end{array}$} \\
\hline & & & Sem & Com & & & & & Sem & Com & & \\
\hline \multirow{3}{*}{ Mucosa $(\mu \mathrm{m})$} & & Sem & 812 & 888 & 850 & & \multirow[b]{3}{*}{1042} & Sem & 1044Ba & $1161 \mathrm{Aa}$ & 1102 & \\
\hline & & Com & 852 & 811 & 832 & \multirow[b]{2}{*}{1,83} & & Com & $1240 \mathrm{Aa}$ & $1057 \mathrm{Ab}$ & 1149 & \\
\hline & 944 & Média & 832 & 850 & & & & Média & 1142 & 1109 & & 1,53 \\
\hline \multirow{3}{*}{ Submucosa $(\mu \mathrm{m})$} & & Sem & 47 & 40 & 43 & \multirow[b]{3}{*}{8,52} & \multirow[b]{3}{*}{72} & Sem & 81 & 83 & 82 & \\
\hline & & Com & 46 & 50 & 48 & & & Com & 81 & 85 & 83 & \\
\hline & 46 & Média & 47 & 45 & & & & Média & 81 & 84 & & 4,17 \\
\hline \multirow{3}{*}{ Muscular circular $(\mu \mathrm{m})$} & & Sem & 226 & 181 & 204 & \multirow[b]{3}{*}{6,07} & \multirow[b]{3}{*}{348} & Sem & 349 & 381 & 365 & \\
\hline & & Com & 197 & 194 & 196 & & & Com & 386 & 341 & 364 & \\
\hline & 204 & Média & 212 & 188 & & & & Média & 367 & 361 & & 2,76 \\
\hline \multirow{3}{*}{ Muscular longitudinal $(\mu \mathrm{m})$} & & Sem & 73 & 62 & 68 & \multirow[b]{3}{*}{8,52} & \multirow[b]{3}{*}{124} & Sem & 129 & 140 & 134 & \\
\hline & & Com & 76 & 77 & 77 & & & Com & 146 & 138 & 142 & \\
\hline & 68 & Média & 75 & 70 & & & & Média & 137 & 139 & & 5,14 \\
\hline \multirow{3}{*}{ Superfície de absorção $\left(\mathrm{mm}^{2}\right)$} & \multirow{3}{*}{0,34} & Sem & 0,056 & 0,065 & 0,061 & \multirow{3}{*}{$\begin{array}{l}0,20 \mathrm{i} \\
19,70\end{array}$} & \multirow[b]{3}{*}{0,074} & Sem & $0,068 \mathrm{Ba}$ & $0,085 \mathrm{Aa}$ & 0,077 & \\
\hline & & Com & 0,058 & 0,054 & 0,056 & & & Com & $0,100 \mathrm{Aa}^{*}$ & $0,064 \mathrm{Ab}$ & 0,082 & \\
\hline & & Média & 0,057 & 0,060 & & & & Média & 0,084 & 0,075 & & 21,80 \\
\hline
\end{tabular}

$\mathrm{CP}=$ controle positivo.

$\mathrm{CV}=$ coeficiente de variação obtido com médias transformadas (log X).

* Média diferente da média do tratamento com antibióticos pelo teste Tukey.

Médias seguidas por letras diferentes maiúsculas nas colunas e minúsculas nas linhas diferem entre si pelo teste Tukey. 
$\mathrm{A}$ adição de $\mathrm{CE}$ às dietas aumentou $(\mathrm{P}<0,04)$ a área de absorção no jejuno aos 42 dias de idade devido a menor quantidade de substratos disponíveis para o crescimento bacteriano que, geralmente, é alto nas porções distais do intestino delgado. Gilbert et al. (2001) notaram que o uso de protease, xilanase e $\alpha$-amilase aumentaram a área de superfície de vilos e Mathlouthi et al. (2002) observaram que a altura das vilosidades aumentou com o uso de xilanase e $\beta$-glucanase. Estes resultados foram atribuídos à menor concentração de bactérias intestinais nas aves alimentadas com dietas contendo as enzimas

Concluiu-se que a adição de MOS às dietas aumentou as espessuras da muscular longitudinal e da mucosa, o que se refletiu em maior superfície de absorção no intestino delgado de frangos de corte aos 21 e 42 dias.

\section{Agradecimentos}

A pesquisa foi financiada pela Fundação de Apoio à Pesquisa do Estado de São Paulo (FAPESP), Alltech do Brasil Agrodindustrial Ltda e Fundação para o Desenvolvimento da Unesp (FUNDUNESP).

\section{Referências}

Brenes, A.; Marquardt, R. R.; Guenter, W.; Viveros, A. 2002. Effect of enzyme addition on the performance and gastrointestinal tract size of chicks fed lupin seed and their fractions. Poultry Science, 81 (5): 670-678.

Chiou, P. W. S.; Yu, B.; Lin, C. 1994. Effect of different components of dietary fiber on intestinal morphology of domestic rabbits. Comparative Biochemistry and Physiology, 108A (4): 262-638.

Edens, F. W.; Parkhurst, C. R.; Casas, I. A.; Dobrogosz, W. J. 1997. Principles of ex ovo competitive exclusion and in ovo administration of Lactobacillus reuteri. Poultry Science, 76 (1): 179-196.

Ferket, P. R. 2004. Alternatives to antibiotics in poultry production: responses, practical experience and recommendations. Proceedings of Alltech`s Annual Symposium, Lexington, USA, p. 54-67.

Ferket, P. R.; Parks, C. W.; Grimes, J. L. 2002. Benefits of dietary antibiotic and mannanoligosaccharide supplementation for poultry. Proceedings of Multi-State Poultry Feeding and Nutrition Conference, Indianapolis, USA, p. 14-16.

Flickinger, E. A.; van Loo, J.; Fahey Jr, G. C. 2003. Nutritional responses to the presence of inulin and oligofructose in the diets of domesticated animals: a review. Critical Review in Food Science and Nutrition, 43 (1): 19-60.
Gilbert, C.; Acamovic, T.; Bedford, M. R. 2001. The effects of lupin inclusion with or without enzyme supplementation on the morphology of the posterior gastrointestinal tract. British Poultry Science, 42 (Supp. 1): S90.

Gracia, M. I.; Araníbar, M. J.; Lázaro, R.; Medel, P.; Mateos, G. G. 2003. $\alpha$-Amylase supplementation of broiler diets based on corn. Poultry Science, 82 (3): 436-442.

Hardin, J. A.; Chung, B.; Loughlin, E. V. O.; Gall, D. G. 1999. The effect of epidermal growth factor on brush border surface area and function in the distal remnant following resection in the rabbit. Gut, 44 (1): 26-32.

Iji, P. A.; Khumalo, K.; Slippers, S.; Gous, R. M. 2003. Intestinal function and body growth of broiler chickens on diets based on maize dried at different temperatures and supplemented with a microbial enzyme. Reproduction Nutrition Development, 43 (1): 77-90.

Jacobs, L. R.; Lupton, J. R. 1984. Effect of dietary fibers on rat large bowel mucosal growth and cell proliferation. American Journal of Physiology, 246 (4): G378-G385.

Kocher, A.; Choct, M.; Ross, G.; Broz, J.; Chung, T.K. 2003. Effects of enzyme combinations on apparent metabolizable energy of corn-soybean meal-based diets in broilers. Journal of Applied Poultry Research, 12 (3): 275-283.

Lan, Y. 2004. Gastrointestinal health benefits of soy water-soluble carbohydrates in young broiler chickens. Tese de Doutorado, Wageningen University, Holanda, 275pp.

Mateos, G. G.; Gonzáles-Alvarado, J. M.; Lázaro, R. 2004. Facing the realities of poultry health and performance without antibiotics in Europe. Proceedings of International Feed Industry Symposium, Lexington, USA, p.69-79.

Mathew, A. G. 2001. Nutritional influences on gut microbiology and enteric diseases. Proceedings of Alltech`s Annual Symposium, Lexington, USA, p. 49-64.

Mathlouthi, N.; Mallet, S.; Saulnier, L.; Quemener, B; Larbier, M. 2002. Effects of xylanase and $\beta$-glucanase addition on performance, nutrient digestibility, and physico-chemical conditions in the small intestine contents and caecal microflora of broiler chickens fed a wheat and barley-based diet. Animal Research, 51 (5): 395-406.

McDonald, D. E.; Pethick, D. W.; Mullan, B. P.; Hampson, D. J. 2001. Increasing viscosity of the intestinal contents alters small intestinal structure and intestinal growth, and stimulates proliferation of enterotoxigenic Escherichia coli in newly-weaned pigs. British Journal of Nutrition, 86 (4): 487-498.

Oliveira, M. C.; Cancherini, L. C.; Gravena, R. A.; Rizzo, P. V.; Moraes, V. M. B. 2007. Utilização de nutrientes de dietas contendo mananoligossacarídeo e/ou complexo enzimático para frangos de corte. Revista Brasileira de Zootecnia, 36 (4): 825-831.

Oliveira, M. C.; Rodrigues, E. A.; Marques, R. H.; Gravena, R. A.; Guandolini, G. C.; Moraes, V. M. B. 2008. Performance and morphology of intestinal mucosa of broilers fed mannan-oligosaccharides and enzymes. Arquivo Brasileiro de Medicina Veterinária e Zootecnia, 60 (2): 442-448.

Rostagno, H. S.; Albino, L. F. T.; Donzele, J. L.; Gomes, P. C.; Ferreira, A. S.; Oliveira, R. F.; Lopes, D. C. 2000. Tabelas brasileiras para aves e suínos. Composição de alimentos e exigências nutricionais. UFV, Viçosa, Brasil, 141pp. 
Santos Jr, A. A.; Ferket, P. R.; Grimes, J. L.; Edens, F. W. 2004. Dietary pentosanase supplementation of diets containing different qualities of wheat on growth performance and metabolizable energy of turkey poults. International Journal of Poultry Science, 3 (1): 33-45.

Statsoft. 1995. Statistica for Windows (Computer program manual). University of Tulsa, USA, CD-Rom.

Schwarz, K. K.; Franco, S. G.; Fedalto, L. M.; Borges, S. A.; Silva, A. V. F.; Pedroso, A. C. 2002. Efeitos de antimicrobianos, probióticos, prebióticos e simbióticos sobre o desempenho e morfologia do jejuno de frangos. Anais da Conferência Apinco de Ciência e Tecnologia Avícola, Campinas, Brasil, p. 75.

Shane, S. M. 2001. Mannan oligosaccharides in poultry nutrition: mechanisms and benefits. Proceedings of Alltech's Annual Symposium, Lexington, USA, p. 65-77.
Sklan, D. 2004. Development of digestive and absorptive functions in the intestine of poultry. Proceedings of World's Poultry Congress, Istambul, Turquia, CD-Rom.

Tungland, B. C.; Meyer, D. 2002. Nondigestible oligo- and polysaccharides (dietary fiber): their physiology and role in human health and food. Comprehensive Review in Food Science and Food Safety, 1 (3): 73-92.

Uni, Z.; Noy, Y.; Sklan, D. 1999. Posthatch development of small intestinal function in the poult. Poultry Science, 78 (2): 215-222.

van Leeuwen, P. 2002. Significance of combined nutritional and morphological precaecal parameters for feed evaluation in nonruminants. Tese de Doutorado, Wageningen University, Holanda, $153 \mathrm{pp}$. 